\title{
REVIEW
}

\section{GLUT1 deficiency and other glucose transporter diseases}

\author{
Juan M Pascual, Dong Wang, Beatriz Lecumberri ${ }^{1}$, Hong Yang, Xia Mao, Ru Yang and Darryl C De Vivo \\ Colleen Giblin Laboratories, Neurological Institute of New York, College of Physicians and Surgeons, Columbia University, New York City, NY, USA and \\ ${ }^{1}$ Departmento de Endocrinología, Clínica Puerta de Hierro, Facultad de Medicina, Universidad Autónoma de Madrid, Madrid, Spain \\ (Correspondence should be addressed to D C De Vivo; Email: GiblinLabs@neuro.columbia.edu)
}

\begin{abstract}
We review the three genetically determined disorders of glucose transport across cell membranes. Diseases such as glucose-galactose malabsorption, Fanconi-Bickel syndrome and De Vivo disease (GLUT1 deficiency syndrome (GLUT1DS)) arise from heritable mutations in transporter-encoding genes that impair monosaccharide uptake, which becomes rate-limiting in tissues where the transporters serve as the main glucose carrier systems. We focus in greater detail on De Vivo disease as a prototype of a brain energy failure syndrome, for which the greatest pathophysiological detail is known, but which presents the most therapeutic challenges. The study of these diseases illustrates fundamental aspects of energetic metabolism, while providing the basis for their diagnosis by simple metabolic screening and for their treatment by dietary modification.
\end{abstract}

European Journal of Endocrinology 150 627-633

\section{Introduction}

Transport of water-soluble molecules across tissue barriers has attracted increasing interest since 1952 (1), when Widdas proposed that the transport of glucose across the erythrocyte membrane required a carrier mechanism to facilitate diffusion. Today, it is established that transport and uptake of glucose can be rate-limiting in cells subject to large metabolic demands, as manifested by several disease states involving transport loss of function. In recent decades, two major families of glucose transporters have been discovered $(2,3)$.

One major family are the concentrative $\mathrm{Na}^{+}$/glucose transporters (SGLT), otherwise known as active cotransporters or symporters. These symporters are energized by $\mathrm{H}^{+}$or $\mathrm{Na}^{+}$gradients. Three types of concentrative $\mathrm{Na}^{+}$/glucose transporters have been identified: SGLT1, SGLT2 and SGLT3. SGLT1 (locus 22q13.1) is responsible for glucose absorption from the intestinal tract. SGLT2 (locus 16p11.2, also designated SLC5A2), together with SGLT1, is responsible for glucose absorption in the renal tubules. SGLT2 is also localized to a lesser degree in ileum. SGLT3 (locus 22q12.2-q12.3) is localized to the plasma membrane of enteric neurons and skeletal muscle, and is incapable of functioning as a homo-oligomer (4).

The other major family of glucose transporters is the GLUT gene family. These transporters facilitate passive diffusion of glucose across tissue barriers by energyindependent stereo-specific mechanisms. In the mid1980s, Mueckler and colleagues discovered a protein that facilitated the diffusion of glucose across the epidermis cell membrane (5). Later, Birnbaum et al. made similar observations in the rat brain, and the two groups proceeded to clone and sequence the gene encoding the GLUT1 protein (Fig. 1) (6). Since 1985, additional proteins have been identified that are responsible for the facilitated diffusion of hexoses across tissue barriers (7). These GLUT genes are a subset of genes within a large superfamily of transport facilitators. This superfamily of transport facilitators is designated SLC2A for solute carrier 2A, according to the HUGO Gene Nomenclature Committee $(8-10)$. GLUT6 was identified as a pseudogene, and GLUT7, initially reported to encode a microsomal transporter, later was shown to be a cloning artifact (11). These earlier missteps have been corrected as new information has emerged. The current understanding of this GLUT gene family has been reviewed recently $(2,7,12)$. The GLUT family includes 12 SLC2A genes, numbered 1-12, encoding 12 GLUT proteins. A thirteenth member of the GLUT family is the myoinositol transporter, HMIT1. GLUT1 is highly expressed in erythrocytes and brain, GLUT2 is associated with Fanconi-Bickel syndrome and is discussed in more detail below, GLUT3 maintains the supply of glucose across the neuronal plasma membrane, GLUT4 is the insulin-regulated glucose transporter of adipose tissues, heart muscles and skeletal muscles that is responsible for insulinregulated glucose transport, GLUT5 is highly expressed in intestine, testis and kidney, and GLUT7 expression currently is unknown. The GLUT7 gene is contiguous 


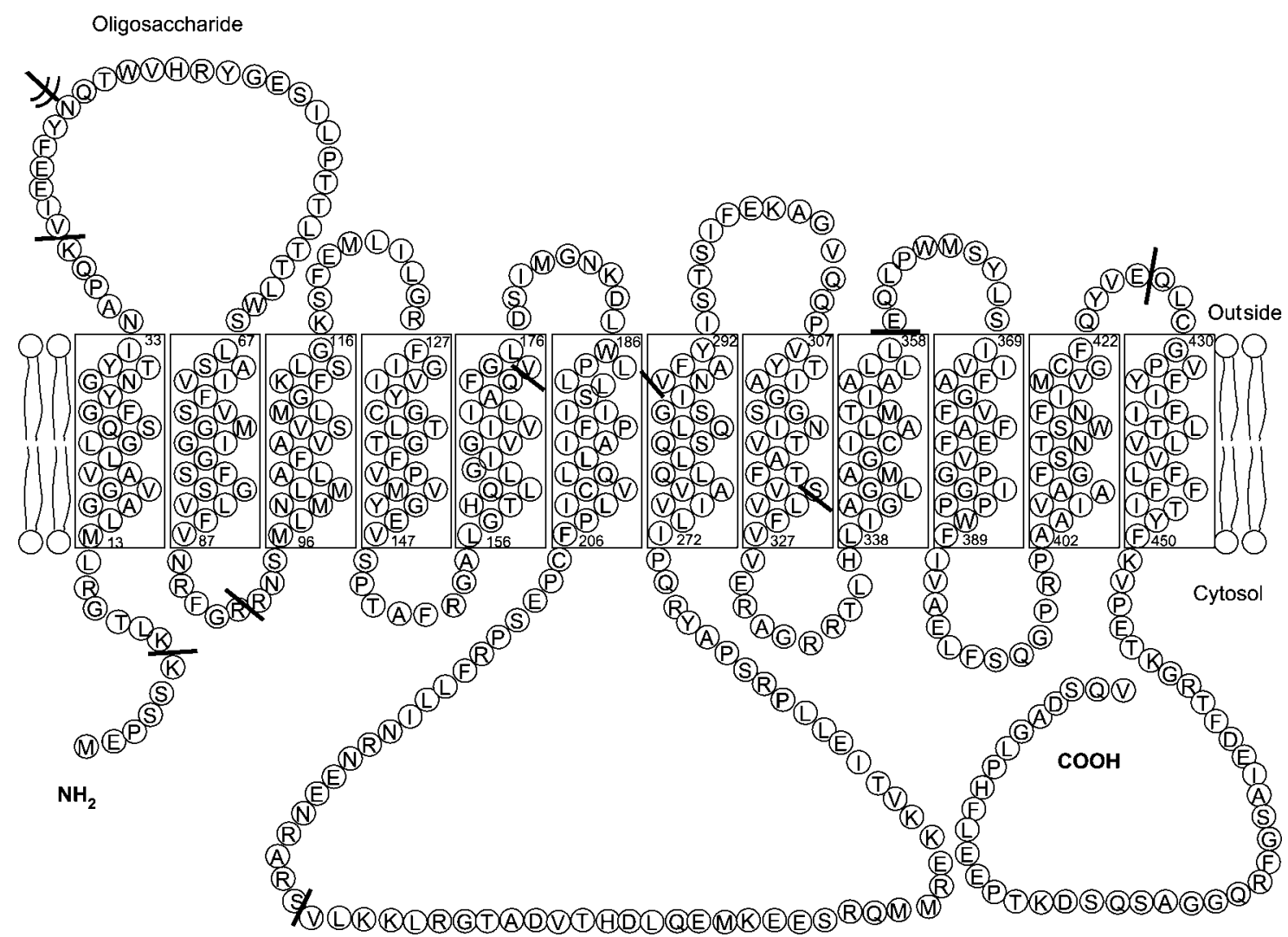

Figure 1 The predicted GLUT1 model. The 12 transmembrane domains are shown in boxes. Solid bars indicate the location of introns in the GLUT1 gene.

to the GLUT5 gene on chromosome 1p36.2 and the GLUT7 protein has a high degree of similarity (58\% identical amino acids) compared with the fructosespecific GLUT5 protein. The GLUT5 protein is known to be present in brain microglia, although its function in these cells remains unclear.

\section{Glucose-galactose malabsorption syndrome (OMIM 606824)}

The glucose-galactose malabsorption syndrome was described in $1962(13,14)$. Both reports, one from Sweden and the other from France, described a familial entity of early onset characterized by watery acid diarrhea and life-threatening dehydration. The symptoms typically begin before the fourth day of life and respond to the elimination of glucose and galactose in the feedings, although they may recur later in life upon reexposure to these monosaccharides. The diagnosis of the disease can be readily confirmed by oral administration of glucose or galactose $(2 \mathrm{~g} / \mathrm{kg})$ followed by lactic acid determination in breath. Fermentation of the heavy monosaccharide contents of the bowel lumen causes the increase in acidity, while water drag accounts for the osmotic diarrhea. All studied patients carry mutations in their Sglt1 genes, while parents are nonmanifesting carriers, speaking to the autosomal recessive pattern of inheritance of this disease. Many patients are the product of consanguineous parents, emphasizing this concept.

\section{Fanconi-Bickel syndrome (OMIM 227810)}

The Fanconi-Bickel syndrome was first described in 1949 (15) and originally named hepatorenal glycogenosis with renal Fanconi syndrome. This first patient presented at age 6 months with failure to thrive, polydipsia and constipation. Later in childhood, he developed osteopenia, short stature, hepatomegaly and tubular nephropathy. The nephropathy was associated with glycosuria, phosphaturia, aminoaciduria and intermittent proteinuria. These urinary metabolites were accompanied by hypophosphatemia and hyperuricemia. The liver was infiltrated with glycogen and fat. Distinctively, ketotic hypoglycemia was evident preprandially, and hyperglycemia was evident postprandially. This distinctive feature is the result of the multifunctional role of GLUT2 in the pancreas and the liver. These patients may exhibit intestinal malabsorption and diarrhea. 
A mutation in the GLUT2 gene was identified in the original case by Santer et al. (16). Most patients with the Fanconi-Bickel syndrome are homozygous for the disease-related mutations consistent with an autosomal recessive pattern of inheritance. Some patients have been shown to be compound heterozygotes (17).

\section{De Vivo disease (GLUT 1DS) (OMIM 606777)}

GLUT1DS was first described in 1991 (18). Since that time, approximately 100 patients have been identified in the USA and elsewhere throughout the world $(2,19-21)$. The clinical syndrome, in and of itself, argues forcefully for the concept that glucose transport across the blood-brain barrier (BBB) is an important rate-limiting event and contributes, in ways not yet fully understood, to impaired early brain growth and functional development. The clinical signature of GLUT1DS is the presence of hypoglycorrhachia and low cerebrospinal fluid (CSF) lactate concentration in the absence of hypoglycemia. Other conditions causing hypoglycorrhachia commonly cause elevated CSF lactate. Examples include mitochondrial diseases, intracranial infection and subarachnoid hemorrhage. Several treatment strategies have been pursued, none optimal, as it relates to the developmental encephalopathy associated with this clinical syndrome. The ketogenic diet has been effective in controlling seizures but has had little measurable effect on the associated cognitive impairment and behavioral disturbances. As the number of cases increases worldwide, it becomes more important to expand our understanding of the clinical phenotypes and to explore alternative treatment strategies designed to mitigate the brain injury associated with impaired glucose transport across the BBB during early neurologic development.

\section{Clinical features}

The phenotypic spectrum of GLUT1DS is becoming broader as more patients are being diagnosed. The clinical signature of the classical phenotype is infantile-onset epileptic encephalopathy associated with delayed neurologic development, deceleration of head growth, acquired microcephaly, incoordination and spasticity. The two children described by De Vivo and colleagues in 1991 exhibited these neurologic signs. The presence of hypoglycorrhachia led the investigators to speculate that there was a defect in the transport of glucose across the BBB. Seven years later, these speculations were substantiated (22). Three clinical phenotypes have been defined with the acquisition of more patients. The first phenotype, the 'classical', is a developmental encephalopathy with seizures. Seizures typically begin within the first 4 months of life. The prenatal and perinatal histories, birth weights and
Apgar scores are normal. The earliest epileptic events include apneic episodes and episodic eye movements simulating opsoclonus. The infantile seizures are clinically fragmented. The frequency of clinical seizures varies considerably from one patient to another. Some have daily seizures whereas others have only occasional seizures separated by days, weeks or months. As a rule, the clinical seizures respond poorly to antiepileptic drugs, and disappear rapidly after patients begin a ketogenic diet.

These patients suffer other paroxysmal events, and it remains unclear whether these events are epileptic or nonepileptic nosologically. Paroxysmal events include intermittent ataxia, confusion, lethargy or somnolence, alternating hemiparesis, abnormalities of movement or posture, total body paralysis, sleep disturbances and recurrent headaches. The frequency of the neurologic symptoms fluctuates unpredictably and may be influenced by environmental factors such as fasting or fatigue (23). The patients have degrees of speech and language impairment. Varying degrees of cognitive impairment have been described, ranging from learning disabilities to severe mental retardation. Acquired microcephaly occurs in $50 \%$ of patients and deceleration of head growth is more common. However, a small number of patients continue to experience normal head growth.

Nonclassical phenotypes also have been identified in recent years. One patient has mental retardation and intermittent ataxia without any clinical seizures (24), and one patient has a movement disorder characterized by choreoathetosis and dystonia. The differential diagnosis has continued to expand as the GLUT1DS phenotypic spectrum has broadened. Several patients have been investigated for occult neuroblastoma because of the opsoclonus-like eye movement abnormalities in early infancy. Other infantile-onset metabolic encephalopathies may be associated with infantile-onset seizures, developmental delay and deceleration of head growth, including chronic hypoglycemic syndromes and disorders of amino acid and organic acid metabolism. Rett syndrome and Angelman syndrome have been considered occasionally, and many patients have been diagnosed initially with cerebral palsy.

\section{Laboratory features}

Examination of the CSF is essentially diagnostic in the correct clinical setting. All patients have hypoglycorrhachia and low normal or low lactate concentrations. This profile is specific for GLUT1DS in the absence of hypoglycemia. The CSF glucose concentrations generally are in the mid-30s $(\mathrm{mg} / \mathrm{dl})$ and seldom, if ever, exceed $40 \mathrm{mg} / \mathrm{dl}$. The CSF lactate values are always lower than $1.4 \mathrm{mM}$.

Brain MRI and CT imaging generally is normal. Minor, nonspecific abnormalities have been described in some patients with slight degrees of brain 
hypotrophy. The PET scan is distinctively abnormal (25). The abnormalities include a global reduction of glucose uptake with more severe hypometabolism in the medial temporal lobes and the thalami. The thalamic hypometabolism is accentuated by the relative uptake of glucose in the basal ganglia.

The erythrocyte glucose uptake study is a valuable screening test and should be carried out in all patients with an appropriate clinical syndrome and associated hypoglycorrhachia (20). The GLUT1 protein in the erythrocyte membrane is immunologically and chemically identical to the GLUT1 isoform in cerebral microvessels (26). The rate of 3-O-methyl-D-glucose uptake by freshly isolated, washed erythrocytes in vitro is decreased in patients compared with controls. The assay is relatively specific and sensitive for GLUT1DS, but false negatives have been observed (27). Chronic hyperglycemic conditions, including diabetes mellitus, also may produce false positives by causing downregulation of GLUT1 receptors in tissue membranes (2).

Fluorescence in situ hybridization (FISH) studies are available and can be performed on blood lymphocytes or cultured skin fibroblasts (22). Three patients with GLUT1DS have been determined to be hemizygous by FISH analysis. This number represents approximately $10 \%$ of patients who have been confirmed at the molecular level.

GLUT1 genomic analysis is indicated in patients who are negative by FISH assay. Genomic DNA can be extracted from the patient's blood, cultured skin fibroblasts or other available tissues. We have identified 30 heterozygous mutations, including 18 missense mutations, three nonsense mutations, three insertions, three microdeletions and three splice-site mutations (Fig. 2).

\section{Biochemistry and metabolism}

Study of GLUT1DS patients has provided clinical proof of the principle that glucose transport across the BBB can be a rate-limiting step for brain metabolism (28). Historically, glucose was considered the obligate fuel for brain metabolism under any and all circumstances. In 1967, ketone bodies were shown to substitute partially for glucose during conditions that favor chronic ketosis (29). Glucose, after entering the brain, is phosphorylated irreversibly to glucose-6-phosphate and metabolized in the pentose phosphate shunt or the Embden-Meyerhoff pathway, or converted to glycogen. The pentose phosphate shunt is important for nucleic acid synthesis. The Embden-Meyerhoff pathway permits glycolytic conversion of glucose to pyruvate. Glycogen synthesis provides a source of fuel during periods of metabolic stress. Decreased entry of glucose into the brain limits these three pathways and potentially contributes to the pathophysiology of GLUT1DS.

Ketone bodies enter the brain by a monocarboxylic transport (MCT) system and are metabolized exclusively

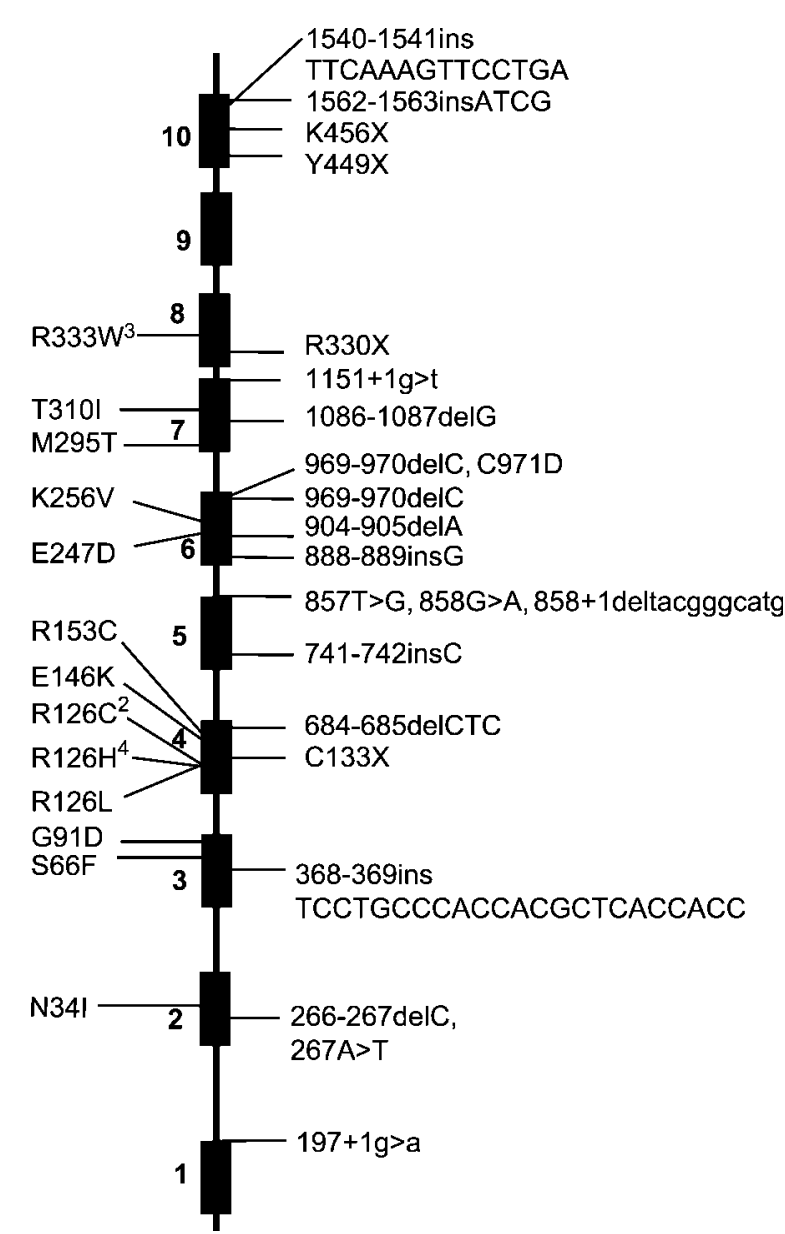

Figure 2 Distribution of identified mutations in GLUT1DS on the GLUT1 gene. The 30 identified mutations currently reported (22, $43-47)$ are shown in the diagram. The three hemizygous mutations are not represented. The 10 exons are shown in filled boxes. The line represents the nine introns, $5^{\prime}$ and $3^{\prime}$ untranslated regions on the GLUT1 gene. The number on the top right corner of the mutation indicates the number of patients carrying the same mutation.

within the mitochondrial matrix to acetyl-CoA (30). Ketone bodies provide an alternative source of acetyl-CoA in conditions that affect pyruvate synthesis from glucose. These conditions include the GLUT1DS, pyruvate dehydrogenase deficiency, and other rare defects of cerebral glycolysis (31).

Magistretti and colleagues have proposed a new theory of brain metabolism by suggesting that lactate, derived from glucose in the astrocyte, is metabolized by the neuron as its principal energy source (32). The Magistretti theory is thought-provoking, but not without its critics (33). Others had shown GLUT3 on the neuronal membrane (34). These issues and interpretations are directly relevant to an understanding of brain metabolism and GLUT1DS pathophysiology. The Magistretti theory suggests that the limitation of glucose transport across the BBB and the astrocyte membrane would limit glycogen storage and synthesis of 
lactate in the astrocyte. This suggestion is consistent with our observations that the CSF lactate is lownormal or low in patients with GLUT1DS. Decreased lactate production by the astrocyte would limit uptake into the neuron, possibly causing secondary energy failure in this brain cell. The development of an animal model will allow these speculations to be investigated directly.

\section{Animal models of glucose transporter diseases}

Several animal models have been developed to assess the pathophysiology of glucose transporter mutations. Homozygous GLUT2 gene disruption causes hyperglycemia and hypoinsulinemia in mice. These animals demonstrate an abnormal glucose tolerance and a loss of insulin control on gene expression by glucose (35). GLUT2 is necessary for maintaining normal glucose homeostasis and normal development and function of the endocrine pancreas. The clinical counterpart for the mouse GLUT2 knockout model is the Fanconi-Bickel syndrome.

A GLUT4 mouse knockout model also has been developed. The female mice exhibit normal blood-glucose concentrations both in the fed and the fasted states, and normal glucose tolerance. The male mice show elevated postprandial blood glucose concentrations compared with control mice. Other glucose transporter isoforms were unchanged in the setting of GLUT4 haploinsufficiency in this gene-targeted knockout mouse model. The fed blood insulin levels in the male and female mice were elevated 5-6-fold, indicating that the GLUT4 knockout mice were insulin resistant. The male mice with heterozygous GLUT4 knockout genotype resemble human beings with non-insulindependent diabetes mellitus. Both the mutant mice and patients with non-insulin-dependent diabetes mellitus have reduced muscle glucose uptake, hypertension and histopathology in the heart and liver (36). GLUT4 knockout mice also had shown reduction in growth rates and shortened life span, possibly resulting from the reported cardiac hypertrophy.

Creation of a GLUT1DS mouse model has been unsuccessful until recently. Heilig has succeeded in creating a transgenic antisense mouse model for GLUT1 haploinsufficiency. His studies indicate that the homozygous mutant mice develop a significant embryopathy with various neurologic malformations, including microcephaly, anencephaly, anophthalmia, and caudal regression (personal communication). These neurologic malformations simulate similar abnormalities in infants of diabetic mothers. A genetargeted homologous knockout mouse model currently is being produced in our laboratory, and the phenotypic manifestations in the heterozygous and homozygous states have yet to be evaluated (Wang, Pascual and De Vivo, unpublished data). These models, in development, will permit investigators to examine the neurochemical, neuropathologic and neurobehavioral consequences of GLUT1 loss of function during fetal and postnatal developments.

\section{Therapy of glucose transporter disorders}

Treatment of glucose-galactose malabsorption includes symptomatic measures during the acute diarrheic phase, followed by the replacement of glucose and galactose in the diet. Fructose may substitute for these carbohydrates.

Fanconi-Bickel syndrome is treated with hydration, electrolytic correction, plasma alkalinization, vitamin $\mathrm{D}$ and phosphate supplementation, restriction of galactose and diabetic diet. Fructose and uncooked cornstarch afford safe carbohydrates that can be utilized for most metabolic needs. However, these interventions have little effect on the rate of somatic growth.

Treatment of De Vivo disease is more challenging. The ketogenic diet was introduced in 1991 as a possible treatment for GLUT1DS before it was confirmed that there was a decrease in glucose transport across tissue barriers (18). Ketone bodies are transported across tissue barriers by monocarboxylic transporters. As a result, ketone bodies are available as an alternative brain fuel source and can be taken up directly into brain cells. The regional distribution of ketone body utilization in the brain is incompletely understood, and the clinical circumstances suggest that the diet is highly effective in controlling the epileptic complications but has no clearly beneficial effect on the behavioral disturbances. Additional studies are necessary to answer this question directly. Transport of glucose across tissue barriers is influenced by other factors. Chronic hyperglycemia causes downregulation of glucose transporters; chronic hypoglycemia or acute hypoxia causes upregulation of GLUT1 transporters. Alpha lipoic acid (thioctic acid) has been shown to facilitate glucose transport in cultured skeletal muscle cells that are GLUT4 dependent (37). Similar, though less convincing studies, have been carried out in GLUT1 transport systems. We recommend, without convincing clinical evidence, that patients take $\alpha$-lipoic acid supplements (38). The patient response has been modest, but the dose taken by mouth may be inadequate to approximate the in vitro corrective effects (39).

Barbiturates are known to inhibit transport of glucose. Most patients with infantile-onset seizures are treated with phenobarbital as the most commonly used antiepileptic drug. Parents have reported anecdotally that phenobarbital not only fails to improve seizure control, but also may worsen their child's neurobehavioral state. We have shown that in vitro barbiturates aggravate the GLUT1 transport defect in erythrocytes from patients with GLUT1DS, and other anticonvulsant effects have been noted as well 
(40, 41). We have made similar observations with methylxanthines, also known to inhibit transport of glucose by GLUT1 (42). One patient, described earlier, reported to us that she was unduly sensitive to coffee and had avoided this beverage since childhood (43). We therefore advise patients to avoid barbiturates, coffee and other caffeine-containing beverages.

All infants and young children are placed on a ketogenic diet as soon as GLUT1DS is suspected. The more definitive laboratory studies can be carried out after the ketogenic diet is started without obscuring the results. Older children and adults become increasingly noncompliant, and some have chosen to resume a regular diet even though their symptoms relapse and seizures recur.

Alternative dietary regimens also need to be explored, including a high-carbohydrate diet fortified with uncooked cornstarch to maintain the blood-glucose concentrations in a higher range. Pharmacologic agents also may be helpful in achieving this goal. For example, diazoxide is a known inhibitor of pancreatic beta-cell insulin release. Diazoxide opens the sulfonylurea receptor-potassium channel complex, resulting in islet cell hyperpolarization and inhibition of insulin release. Glucagon also raises blood glucose by stimulating gluconeogenesis and glycogenolysis, and by inhibiting glucose uptake by hepatic and muscle tissues. Diazoxide may prove helpful as a daily treatment to raise the interprandial blood glucose concentrations, and glucagon may prove helpful in treating acute paroxysmal events.

\section{References}

1 Widdas WF. Inability of diffusion to account for placental glucose transfer in the sheep and consideration of kinetics of a possible carrier transfer. Journal of Physiology 1952118 23-29.

2 De Vivo DC, Wang D, Pascual JM \& Ho YY. Glucose transporter protein deficiency syndromes. In International Review of Neurobiology, vol 51, pp 259-288. Ed. DS Dwyer. San Diego, CA: Academic Press, 2002.

3 Joost HG, Bell GI, Best JD, Birnbaum MJ, Charron MJ, Chen YT, Doege H, James DE, Lodish HF, Moley KH, Moley JF, Mueckler M, Rogers S, Schurmann A, Seino S \& Thorens B. Nomenclature of the GLUT/SLC2A family of sugar/polyol transport facilitators. American Journal of Physiology, Endocrinology and Metabolism 2002282 E974-E976.

4 Diez-Sampedro A, Hirayama BA, Osswald C, Gorboulev V, Baumgarten K, Volk C, Wright EM \& Koepsell H. A glucose sensor hiding in a family of transporters. PNAS $2003 \mathbf{1 0 0}$ 11753-11758.

5 Mueckler M, Caruso C, Baldwin SA, Panico M, Blench I, Morris HR, Allard WJ, Lienhard GE \& Lodish HF. Sequence and structure of a human glucose transporter. Science 1985229 941-945.

6 Birnbaum MJ, Haspel HC \& Rosen OM. Cloning and characterization of a cDNA encoding the rat brain glucose-transporter protein. PNAS $1986 \mathbf{8 3} 5784-5788$.

7 Joost HG \& Thorens B. The extended GLUT-family of sugar/polyol transport facilitators: nomenclature, sequence characteristics, and potential function of its novel members. Molecular Membrane Biology 200118 247-256.
8 Marger MD \& Saier MH Jr. A major superfamily of transmembrane facilitators that catalyse uniport, symport and antiport. Trends in Biochemical Sciences $19931813-20$.

9 Saier MH Jr, Beatty JT, Goffeau A, Harley KT, Heijne WH, Huang SC, Jack DL, Jahn PS, Lew K, Liu J, Pao SS, Paulsen IT, Tseng TT \& Virk PS. The major facilitator superfamily. Journal of Molecular Microbiology and Biotechnology 19991 257-279.

10 Sauer N \& Stadler R. A sink-specific $\mathrm{H}+/$ monosaccharide co-transporter from Nicotiana tabacum: cloning and heterologous expression in baker's yeast. Plant Journal 1993 4 601-610.

11 Burchell A. A re-evaluation of GLUT 7. Biochemical Journal 1998 331973.

12 Ho Y-Y, Wang D \& De Vivo D. Glucose transporters. Wiley Encyclopedia of Molecular Medicine $200151441-1446$.

13 Lindquist B \& Meeuwisse G. Chronic diarrhoea caused by monosaccharide malabsorption. Acta Paediatrica $196251674-685$.

14 Laplane R, Polonovoski C, Etienne M, Debray P, Lods J-C \& Pissarro B. L'intolerance aux sucres a transfert intestinal actif. Archives of French Pediatrics 196219 895-944.

15 Fanconi G \& Bickel H. Die chronische aminoacidurie (Aminosaeurediabetes oder nephrotisch-glukosurisscher Zwergwuchs) ber der Glykogenose und Cystinkrankheit. Helvetica Paediatrica Acta $19494359-396$.

16 Santer R, Schneppenheim R, Dombrowski A, Gotze H, Steinmann B \& Schaub J. Mutations in GLUT2, the gene for the liver-type glucose transporter, in patients with Fanconi-Bickel syndrome. Nature Genetics $199717324-326$.

17 Santer R, Groth S, Kinner M, Dombrowski A, Berry GT, Brodehl J, Leonard JV, Moses S, Norgren S, Skovby F, Schneppenheim R, Steinmann B \& Schaub J. The mutation spectrum of the facilitative glucose transporter gene SLC2A2 (GLUT2) in patients with Fanconi-Bickel syndrome. Human Genetics $200211021-29$.

18 De Vivo DC, Trifiletti RR, Jacobson RI, Ronen GM, Behmand RA \& Harik SI. Defective glucose transport across the blood-brain barrier as a cause of persistent hypoglycorrhachia, seizures, and developmental delay. New England Journal of Medicine 1991325 703-709.

19 Klepper J \& Voit T. Facilitated glucose transporter protein type 1 (GLUT1) deficiency syndrome impaired glucose transport into brain - a review. European Journal of Pediatrics 2002161 295-304.

20 Pascual JM, Wang D \& De Vivo DC. Glucose transporter type 1 deficiency syndrome. De Vivo disease GeneReviews. Database online at www.geneclinics.org, 2002.

21 De Vivo D, Wang D \& Pascual JM. Disorders of glucose transport. In The Molecular and Genetic Basis of Neurologic and Psychiatric Disease, 3rd edn, pp 625-634. Eds RN Rosenberg, SB Prusiner, S DiMauro, RL Barchi \& EJ Nestler. Philadelphia: Butterworth-Heinemann, 2003.

22 Seidner G, Alvarez MG, Yeh JI, O’Driscoll KR, Klepper J, Stump TS, Wang D, Spinner NB, Birnbaum MJ \& De Vivo DC. GLUT-1 deficiency syndrome caused by haploinsufficiency of the bloodbrain barrier hexose carrier. Nature Genetics $199818188-191$.

23 von Moers A, Brockmann K, Wang D, Korenke CG, Huppke P, De Vivo DC \& Hanefeld F. EEG features of glut-1 deficiency syndrome. Epilepsia $2002 \mathbf{4 3} 941-945$.

24 Overweg-Plandsoen W, Groener JE, Wang D, Onkenhout W, Brouwer OF, Bakker HD \& De Vivo DC. GLUT-1 deficiency without epilepsy - an exceptional case. Journal of Inherited Metabolism Disorders 200326 559-563.

25 Pascual JM, Van Heertum RL, Wang D, Engelstad K \& De Vivo DC. Imaging the metabolic footprint of Glut1 deficiency on the brain. Annals of Neurology $2002 \mathbf{5 2} 458-464$.

26 Kalaria RN, Gravina SA, Schmidley JW, Perry G \& Harik SI. The glucose transporter of the human brain and blood-brain barrier. Annals of Neurology $1988 \mathbf{2 4} 757-764$.

27 Klepper J, Garcia-Alvarez M, O’Driscoll KR, Parides MK, Wang D, Ho YY \& De Vivo DC. Erythrocyte 3-O-methyl-D-glucose uptake assay for diagnosis of glucose-transporter-protein syndrome. Journal of Clinical Laboratory Analysis 199913 116-121. 
28 Lund-Andersen H \& Kjeldsen CS. Uptake of glucose analogues by rat brain cortex slices membrane transport versus metabolism of 2-deoxy-D-glucose. Journal of Neurochemistry 197729 205-211.

29 Owen OE, Morgan AP, Kemp HG, Sullivan JM, Herrera MG \& Cahill GF Jr. Brain metabolism during fasting. Journal of Clinical Investigation $1967 \mathbf{4 6} 1589-1595$.

30 De Vivo DC. The effects of ketone bodies on glucose utilization. In Cerebral Metabolism and Neural Function, pp 243-254. Eds JV Passonneau, RA Hawkins \& RA Lust. Baltimore, MD: Williams \& Wilkins, 1980

31 De Vivo DC, Leary L \& Wang D. Glut-1 deficiency syndrome and other glycolytic defects. Journal of Child Neurology 200217 515-523.

32 Magistretti PJ. Cellular bases of functional brain imaging insights from neuron-glia metabolic coupling. Brain Research $2000 \mathbf{8 8 6}$ 108-112.

33 Chih CP, Lipton P \& Roberts EL Jr. Do active cerebral neurons really use lactate rather than glucose? Trends in Neurosciences $200124573-578$.

34 Vannucci SJ, Maher F \& Simpson IA. Glucose transporter proteins in brain delivery of glucose to neurons and glia. Glia $1997 \mathbf{2 1}$ $2-21$.

35 Guillam MT, Hummler E, Schaerer E, Yeh JI, Birnbaum MJ, Beermann F, Schmidt A, Deriaz N, Thorens B \& Wu JY. Early diabetes and abnormal postnatal pancreatic islet development in mice lacking Glut-2. Nature Genetics 199717 327-330.

36 Stenbit AE, Tsao TS, Li J, Burcelin R, Geenen DL, Factor SM, Houseknecht K, Katz EB \& Charron MJ. GLUT4 heterozygous knockout mice develop muscle insulin resistance and diabetes. Nature Medicine 19973 1096-1101.

37 Klip A, Tsakiridis T, Marette A \& Ortiz PA. Regulation of expression of glucose transporters by glucose: a review of studies in vivo and in cell cultures. FASEB Journal 19948 43-53.

38 De Vivo DC, Garcia AM \& Tristschler HJ. Deficiency of glucose transporter protein type 1: possible therapeutic role for alphalipoic acid (thioctic acid). Diabetes und Stoffwechsel $1996 \mathbf{5}$ 36-40.

39 Kulikova-Schupak R, Ho YY, Kranz-Eble P, Yang H, Wang D \& De Vivo DC. Stimulation of GLUT-1 gene transcription by thioctic acid and its potential therapeutic value in Glut-1 deficiency syndrome (GLUT-1DS). Journal of Inherited Metabolic Disease 2001 $24 \mathrm{~S} 106$.

40 Klepper J, Fischbarg J, Vera JC, Wang D \& De Vivo DC. GLUT1deficiency barbiturates potentiate haploinsufficiency in vitro. Pediatric Research 199946 677-683.

41 Klepper J, Schaper J, Raca G, Coryell J, Das S, Hayflick SJ \& Voit T. Effects of anticonvulsants on GLUT1-mediated glucose transport in GLUT1 deficiency syndrome in vitro. European Journal of Pediatrics $200316284-89$.

42 Ho YY, Yang H, Klepper J, Fischbarg J, Wang D \& De Vivo DC. Glucose transporter type 1 deficiency syndrome (Glut1DS) methylxanthines potentiate GLUT1 haploinsufficiency in vitro. Pediatric Research 200150 254-260.

43 Brockmann K, Wang D, Korenke CG, von Moers A, Ho YY, Pascual JM, Kuang K, Yang H, Ma L, Kranz-Eble P, Fischbarg J, Hanefeld F \& De Vivo DC. Autosomal dominant glut-1 deficiency syndrome and familial epilepsy. Annals of Neurology $2001 \mathbf{5 0}$ 476-485.

44 Klepper J, Wang D, Fischbarg J, Vera JC, Jarjour IT, O’Driscoll KR \& De Vivo DC. Defective glucose transport across brain tissue barriers: a newly recognized neurological syndrome. Neurochemical Research 199924 587-594.

45 Wang D, Kranz-Eble P \& De Vivo DC. Mutational analysis of GLUT1 (SLC2A1) in Glut-1 deficiency syndrome. Human Mutation $200016224-231$.

46 Klepper J, Willemsen M, Verrips A, Guertsen E, Herrmann R, Kutzick C, Florcken A \& Voit T. Autosomal dominant transmission of GLUT1 deficiency. Human Molecular Genetics 2001 10 63-68.

47 Lange P, Gertsen E, Monden I, Klepper J \& Keller K. Functional consequences of an in vivo mutation in exon 10 of the human GLUT1 gene. FEBS Letters $2003 \mathbf{5 5 5} 274-278$.

Received 10 December 2003

Accepted 11 February 2004 